\title{
ÁRVORES, PALMEIRAS E CICAS DE QUATRO PRAÇAS DO MUNICÍPIO DE JAÚ, SP
}

\author{
TREES, PALM TREES AND CICAS OF FOUR SQUARES OF JAÚ MUNICIPALITY, SP \\ Bruna Letícia da Matta ${ }^{1}$, Frederico Fregolente Faracco Mazziero², Ricardo Kutschinsky Bastos³, \\ Ricardo da Silva Oliveira ${ }^{4}$, Natália Arias Galastri ${ }^{5}$
}

\section{RESUMO}

A arborização urbana exerce um papel fundamental no contexto urbano, podendo trazer diversos benefícios, mas não tem sido encarada como prioridade na maioria das cidades, que não apresentam planejamento de sua arborização. Dessa maneira, o presente estudo objetivou inventariar as espécies de árvores, palmeiras e cicas encontradas em quatro praças localizadas no Município de Jaú, além de averiguar a origem de cada uma, suas abundâncias e frequências. Para isso, todos os indivíduos arbóreos encontrados nas Praças Francisco Pinto, João Paulo II, Ângelo Grizzo e da Escola Técnica Joaquim Ferreira do Amaral foram inventariados durante os anos de 2012 a 2016. Foram encontrados 760 indivíduos de 107 espécies distribuídas em 38 famílias e 85 gêneros. As famílias Fabaceae, Myrtaceae, Arecaceae, Bignoniaceae e Moraceae foram as mais representativas, correspondendo juntas a $48,4 \%$. As quatro praças surpreenderam quanto ao número de espécies, bem maior que o encontrado em vários outros estudos. Dentre as espécies encontradas $46 \%$ são nativas do Brasil enquanto $54 \%$ são exóticas, números condizentes com os resultados mais equilibrados observados em outros estudos. Portanto, a riqueza de espécies utilizadas na arborização destas praças de Jaú pode ser considerada elevada e o grande número de espécies exóticas pode trazer problemas.

Palavras-chave: Árvores; Riqueza; Áreas Verdes; Inventário; Fabaceae.

\section{ABSTRACT}

Urban forestry has a fundamental role in the urban context, which can bring several benefits, but it has not been considered a priority in most cities, which do not present planning of their urban forestry. Thus, the aim of this study was to inventory the trees, palm trees and cicas species found in four squares located in the municipality of Jaú, as well as to ascertain the origin of each one, its abundances and frequencies. For this, all individuals of tree species found in the squares Francisco Pinto, João Paulo II, Ângelo Grizzo and in the Technical School Joaquim Ferreira do Amaral were inventoried during the years 2012 to 2016. A total of 760 individuals of 107 species, distributed in 38 families and 85 genera, were found. The families Fabaceae, Myrtaceae, Arecaceae, Bignoniaceae and Moraceae were the most representative, corresponding together to $48.4 \%$. The four squares surprised in the number of species, much larger than that found in several other studies. Among the species found, $46 \%$ are native to Brazil, while $54 \%$ are exotic, numbers consistent with the more balanced results observed in other studies. Therefore, the richness of species used in the afforestation of these Jaú squares can be considered high and the large number of exotic species can cause problems.

Keywords: Trees; Species richness; Green areas; Inventory; Fabaceae.

Recebido em 06.05.2017 e aceito em 22.09.2017

1 Graduanda em Tecnologia em Meio Ambiente e Recursos Hídricos. FATEC JAHU.Jaú/SP.Email: bruhbruhdamatta@hotmail.com 2 Biólogo. Mestre em Botânica. Graduando em Tecnologia em Meio Ambiente e Recursos Hídricos. FATEC JAHU. Jaú/SP. Email: fredericobio2@gmail.com

3 Graduando em Tecnologia em Meio Ambiente e Recursos Hídricos. FATEC JAHU. Jaú/SP. Email: ricardokbastos@gmail.com 4 Graduando em Tecnologia em Meio Ambiente e Recursos Hídricos. FATEC JAHU. Jaú/SP. Email: rick_friend182@hotmail.com 5 Bióloga. Doutora em Biologia Vegetal. Professora da Faculdade de Tecnologia de Jahu. Jaú/SP. Email: natalia.galastri@fatec.sp.gov.br 


\section{INTRODUÇÃO}

A preocupação com a arborização urbana é um item relativamente novo dentro das atribuições do poder público brasileiro, mesmo ela remontando há mais de 120 anos. De acordo com Silva, Silveira e Teixeira (2008), a ausência de planejamento na arborização urbana tem gerado situações que impossibilitam a padronização dos processos envolvidos na arborização, fazendo com que essa fique irregular, o que, no futuro, pode ocasionar problemas e inconvenientes. Estes problemas estão relacionados a plantios irregulares, feitos por pessoas sem o devido conhecimento, e aos danos estruturais ao patrimônio urbano público e privado como quebra de calçadas e tubulações subterrâneas e problemas com a fiação.

Apesar disso, as árvores no meio urbano são de extrema importância, pois trazem diversos benefícios à população local, intervindo diretamente e de forma positiva nas condições climáticas, no bem estar e saúde da população e na estética local.

Para isso, segundo Coleto, Müller e Wolski (2008), o planejamento da arborização urbana é essencial e deve ser feito através de inventários quali-quantitativos que mostram a situação atual. Portanto, estes inventários servem de diretrizes que poderão ser utilizadas pelo poder público para saber quais medidas devem ser tomadas, buscando, ao máximo, usufruir dos benefícios gerados pela arborização urbana.

As praças são espaços públicos em meio à área urbana que desempenham importantes funções às cidades e, principalmente, aos cidadãos, pois propiciam lazer, convívio social, lembranças, além do contato com a natureza, o que, em muitas cidades, só acontece por meio desses espaços (ROBBA; MACEDO, 2010).

As praças também trazem diversos outros benefícios, como a diminuição da poluição sonora, a melhora no microclima local e na qualidade do ar, além de aumentar a biodiversidade e de produzir alimento e fornecer abrigo para os animais presentes nestas áreas (ROBBA; MACEDO, 2010).

A cidade de Jaú apresenta várias praças, dentre as quais as Praças Francisco Pinto, João Paulo II, Ângelo Grizzo e da Escola Técnica Joaquim Ferreira do Amaral que se destacam pela proximidade com o centro da cidade, pela frequência que são utilizadas pela população e pela história, uma vez que são praças bastante antigas.

Diante disso, o objetivo do presente estudo foi realizar o levantamento das espécies de árvores, palmeiras e cicas encontradas nessas quatro praças localizadas no Município de Jaú, interior de São Paulo, além de averiguar a origem de cada uma, suas abundâncias e frequências. 


\section{MATERIAL E MÉTODOS}

As coletas foram realizadas em quatro praças do Município de Jaú, Estado de São Paulo, cujo clima pode ser enquadrado, segundo a classificação de Köppen, em "Cwa" (ALVARES et al., 2014), ou seja, apresenta inverno frio e seco, com verão quente e chuvoso.

O levantamento foi realizado na porção central do município de Jaú e para a seleção das praças, levaram-se em conta os mesmos parâmetros adotados por Carcereri, Biondi e Batista (2016), considerando estes adequados e suficientes para o presente estudo. No entanto, foram feitas adequações, sendo consideradas apenas as praças pertencentes à porção central da cidade e não foi estabelecida uma área mínima, evitando assim que o número total de praças fosse reduzido. Assim, a porção central do município está representada por 18 praças que se enquadram nestes requisitos. Destas, foram sorteadas quatro praças para a amostragem piloto, sendo posteriormente realizada uma análise de intensidade amostral, conforme proposto por Carcereri, Biondi e Batista (2016). Por fim, após esta estimativa, verificou-se que 3,4 praças ou $18,8 \%$ deveriam ser mensuradas e, portanto, as amostras realizadas no piloto foram suficientes para representar as praças desta região do município, uma vez que esta amostra levou em conta $22,2 \%$ do total de praças.

As quatro praças selecionadas (Figura 1) para a realização deste trabalho foram: (1) Praça Francisco Pinto (P1), situada no Bairro Vila Netinho sob as coordenadas UTM 752353/7544909, com 4.676m² de área (Figura 2A); (2) Praça João Paulo II (P2), no Bairro Chácara Bela Vista sob as coordenadas UTM 752629/7583832, com 5.528m² de área (Figura 2B); (3) Praça Ângelo Grizzo (P3), no Bairro Vila Nova nas coordenadas UTM 750127/7531698, com 7.361 $\mathrm{m}^{2}$ de área (Figura 2C); e (4) Praça da Escola Técnica Joaquim Ferreira do Amaral situada nas coordenadas UTM 751068/7532797, com 5.502 $\mathrm{m}^{2}$ de área (Figura 2D). Neste último ponto funciona a escola técnica, sendo que, neste caso, optou-se em amostrar todos os indivíduos da região do entorno e do interior desta, pois apesar da separação por muros, os jardins internos e a praça formam um contínuo da arborização. 


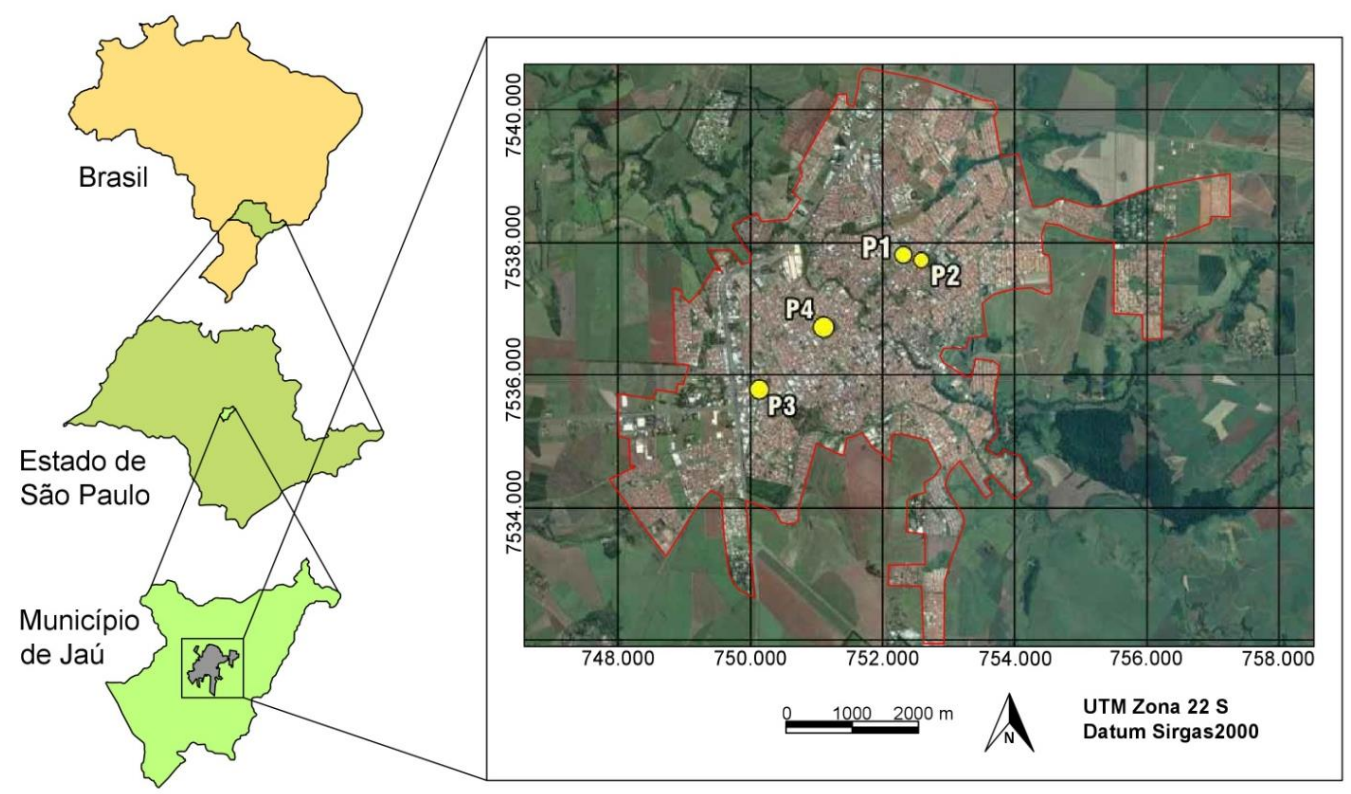

Fonte: Imagem do Google Earth adaptada (data da imagem 17/06/2016)

Figura 1. Localização do município de Jaú no estado de São Paulo evidenciando o perímetro urbano com os quatro pontos de amostragem (P1 = Praça Francisco Pinto; P2 = Praça João Paulo II; P3 = Praça Ângelo Grizzo; P4 = Praça da Escola Técnica Joaquim Ferreira do Amaral; linha vermelha = perímetro urbano)

Figure 1. Location of Jaú in the São Paulo's state showing the urban perimeter with the four points sampled $(\mathrm{P} 1=$ Francisco Pinto Square; P2 = João Paulo II Square; P3 = Ângelo Grizzo Square; P4 = Joaquim Ferreira do Amaral Technical School Square; red line = urban perimeter)
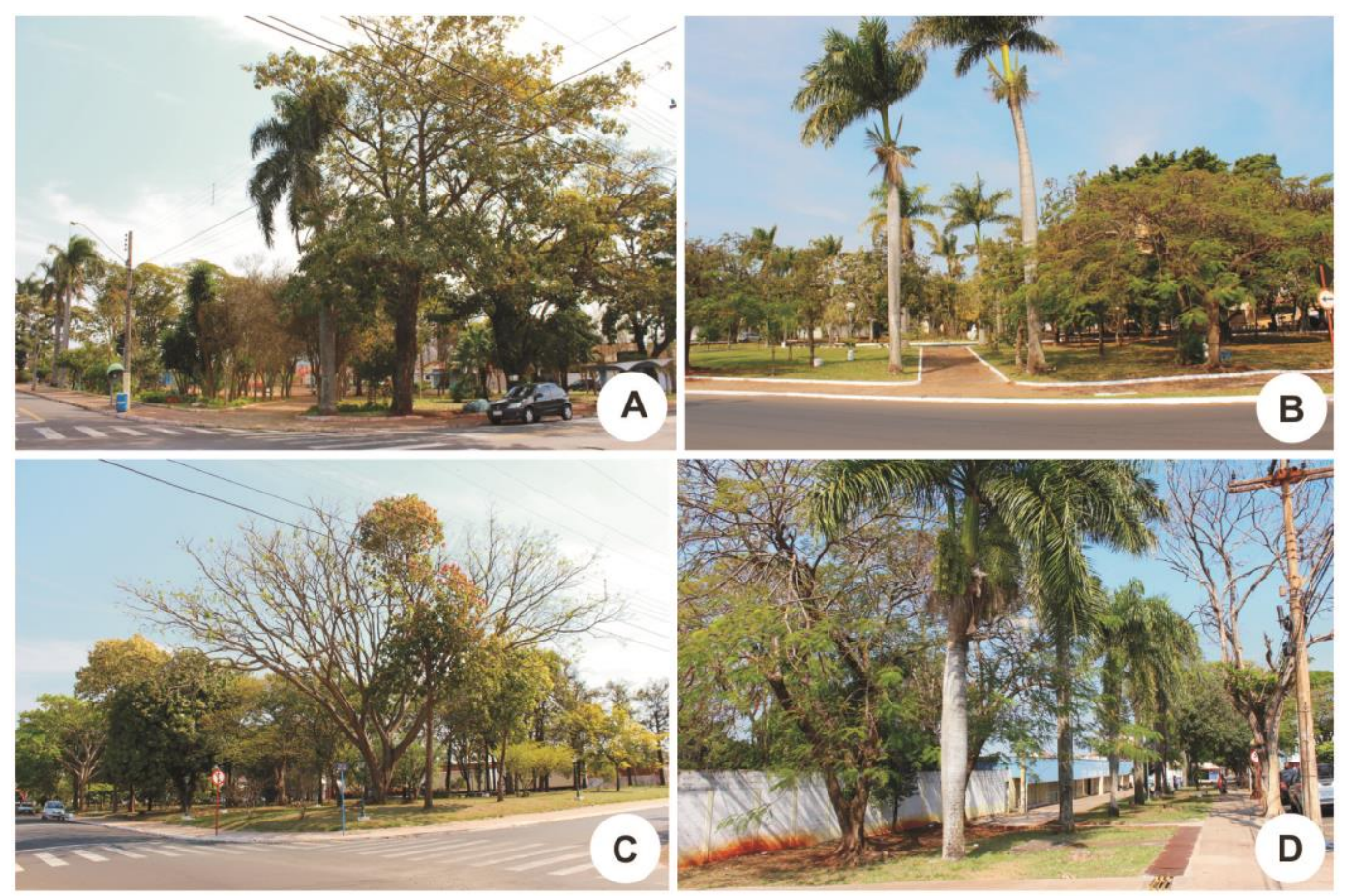

Figura 2. Vista geral das praças estudadas. A. Praça Francisco Pinto (P1). B. Praça João Paulo II (P2). C. Praça Ângelo Grizzo (P3). D. Praça da Escola Técnica Joaquim Ferreira do Amaral (P4)

Figure 2. General view of the squares studied. A. Francisco Pinto Square (P1). B. João Paulo II Square (P2). C. Ângelo Grizzo Square (P3). D. Joaquim Ferreira do Amaral Technical School Square (P4) 
Todos os indivíduos de espécies arbóreas, de palmeiras e de cicas encontrados nessas quatro praças foram levantados. Devido à disponibilidade de recursos humanos e financeiros, a Praça da Escola Técnica Joaquim Ferreira do Amaral foi avaliada durante o ano de 2012, a Praça Ângelo Grizzo durante o ano de 2014, a Praça Francisco Pinto no ano de 2015 e a Praça João Paulo II no ano de 2016. Apenas as espécies cuja identificação não foi possível em campo foram coletadas, principalmente para a realização da comparação do material com a bibliografia específica e acervos disponíveis em herbários ou de forma digital.

O tratamento taxonômico adotado para as angiospermas foi o APG IV (2016) e para gimnospermas Christenhusz et al. (2011). O nome das espécies, bem como a abreviação do nome dos autores dos táxons, segue a Lista de Espécies da Flora do Brasil (2017) para espécies nativas, e Mobot (2017) para espécies consideradas exóticas. Optou-se em considerar nativa do Brasil toda espécie tratada assim pela Lista de Espécies da Flora do Brasil (2017), e exótica toda aquela que não constasse neste banco de dados ou que tenha sido tratada como naturalizada ou subespontânea.

Foram também calculadas a abundância e a frequência de cada espécie, sendo a abundância o número total de indivíduos de cada espécie, e a frequência a relação entre o número de indivíduos da espécie e número total de indivíduos expressa em porcentagem.

\section{RESULTADOS E DISCUSSÃO}

Foram encontrados 760 indivíduos de 107 espécies, distribuídas em 38 famílias e 85 gêneros (Tabela 1). A Praça Francisco Pinto (P1) apresentou 208 indivíduos de 25 famílias e 52 espécies, por sua vez a Praça João Paulo II (P2) obteve 223 indivíduos de 21 famílias e 45 espécies, a Praça Ângelo Grizzo (P3) apresentou 160 indivíduos pertencentes a 20 famílias e 37 espécies e na Praça da Escola Técnica Joaquim Ferreira do Amaral (P4) foram encontrados 169 indivíduos de 19 famílias e 38 espécies (Tabela 1).

Em relação ao número de famílias encontradas em todas as áreas estudadas, o município de Jaú parece se destacar em relação a outros estudados como, por exemplo, no Município de Mata, no Rio Grande do Sul, onde Richter et al. (2012) encontraram, tanto em vias públicas quanto praças, apenas 34 famílias, número inferior ao registrado em apenas quatro praças no presente estudo. 
Tabela 1. Lista das espécies de árvores, palmeiras e cicas encontradas em quatro praças no município de Jaú, São Paulo, Brasil

Table 1. List of trees, palm trees and cicas species found in four squares in the municipality of Jaú, São Paulo, Brazil

\begin{tabular}{|c|c|c|c|c|c|c|c|c|}
\hline Família/Espécie & Nome comum & 0 & P1 & P2 & P3 & P4 & $\mathbf{T}$ & $F(\%)$ \\
\hline \multicolumn{9}{|l|}{ Anacardiaceae } \\
\hline Anacardium occidentale L. & Cajú & $\mathrm{N}$ & 1 & 1 & 1 & - & 3 & 0,39 \\
\hline Mangifera indica L. & Mangueira & $E$ & 9 & 9 & 8 & - & 26 & 3,42 \\
\hline Schinus molle L. & Aroeira-salsa & $\mathrm{N}$ & - & - & - & 1 & 1 & 0,13 \\
\hline Schinus terebinthifolius Raddi & Aroeira-pimenteira & $\mathrm{N}$ & 2 & - & - & 2 & 4 & 0,53 \\
\hline \multicolumn{9}{|l|}{ Annonaceae } \\
\hline Annona squamosa L. & Fruta-do-conde & $E$ & 1 & 1 & - & - & 2 & 0,26 \\
\hline Annona muricata L. & Graviola & $E$ & - & 2 & - & - & 2 & 0,26 \\
\hline \multicolumn{9}{|l|}{ Apocynaceae } \\
\hline Nerium oleander L. & Espirradeira & $E$ & 3 & - & - & - & 3 & 0,39 \\
\hline Plumeria rubra L. & Jasmin-manga & $E$ & 1 & - & - & - & 1 & 0,13 \\
\hline $\begin{array}{l}\text { Tabernaemontana catharinensis A. } \\
\text { DC. }\end{array}$ & Leiteira & $\mathrm{N}$ & 3 & 1 & - & - & 4 & 0,53 \\
\hline $\begin{array}{l}\text { Thevetia thevetioides (Kunth) } \\
\text { K.Schum. } \\
\text { Araliaceae }\end{array}$ & $\begin{array}{l}\text { Chapéu-de- } \\
\text { napoleão }\end{array}$ & $E$ & - & - & 4 & - & 4 & 0,53 \\
\hline Schefflera actinophylla (Endl.) Harms & Cheflera & $E$ & - & - & - & 12 & 12 & 1,58 \\
\hline $\begin{array}{l}\text { Schefflera arboricola (Hayata) Merr. } \\
\text { Araucariaceae }\end{array}$ & Cheflera-pequena & $E$ & - & - & - & 3 & 3 & 0,39 \\
\hline $\begin{array}{l}\text { Araucaria angustifolia (Bertol.) Kuntze } \\
\text { Arecaceae }\end{array}$ & Araucária & $\mathrm{N}$ & - & - & 2 & - & 2 & 0,26 \\
\hline $\begin{array}{l}\text { Acrocomia aculeata (Jacq.) Lodd. ex } \\
\text { Mart. }\end{array}$ & Macaúba & $\mathrm{N}$ & - & - & - & 1 & 1 & 0,13 \\
\hline $\begin{array}{l}\text { Archontophoenix alexandrae } \\
\text { (F.Mueller) H.Wendl. \& Drude }\end{array}$ & Palmeira-beatriz & $E$ & - & 1 & - & - & 1 & 0,13 \\
\hline $\begin{array}{l}\text { Dypsis lutescens (H.Wendl.) Beentje \& } \\
\text { J.Dransf. }\end{array}$ & Areca-bambu & $E$ & 2 & 5 & - & - & 7 & 0,92 \\
\hline Livistona australis Mart. & $\begin{array}{l}\text { Palmeira-leque-de- } \\
\text { saia }\end{array}$ & $E$ & - & - & - & 2 & 2 & 0,26 \\
\hline Livistona chinensis (Jacq.) R. Br. & $\begin{array}{l}\text { Palmeira-leque-da- } \\
\text { china }\end{array}$ & $E$ & 18 & 2 & 3 & - & 23 & 3,03 \\
\hline Phoenix sp. & - & $E$ & - & - & 2 & - & 2 & 0,26 \\
\hline Roystonea borinquena O.F.Cook & $\begin{array}{l}\text { Palmeira-imperial- } \\
\text { de-porto-rico }\end{array}$ & $E$ & - & - & - & 18 & 18 & 2,37 \\
\hline Roystonea oleraceae L.H.Bailey & Palmeira-imperial & $E$ & 9 & 20 & 2 & - & 31 & 4,08 \\
\hline Syagrus oleracea (Mart.) Becc. & Guariroba & $\mathrm{N}$ & - & 1 & - & 2 & 3 & 0,39 \\
\hline $\begin{array}{l}\text { Syagrus romanzoffiana (Cham.) } \\
\text { Glassman } \\
\text { Asparagaceae }\end{array}$ & Jerivá & $\mathrm{N}$ & - & 2 & - & 1 & 3 & 0,39 \\
\hline $\begin{array}{l}\text { Yucca guatemalensis Baker } \\
\text { Bignoniaceae }\end{array}$ & luca-elefante & $E$ & 6 & - & - & - & 6 & 0,79 \\
\hline $\begin{array}{l}\text { Crescentia cujete L. } \\
\text { Handroanthus heptaphyllus (Vell.) }\end{array}$ & Cabaça & $E$ & - & 1 & - & - & 1 & 0,13 \\
\hline Mattos & Ipê-roxo & $\mathrm{N}$ & 3 & - & - & 6 & 9 & 1,18 \\
\hline
\end{tabular}


Handroanthus impetiginosus (Mart. ex DC.) Mattos

Jacaranda cuspidifolia Mart.

Jacaranda mimosifolia D. Don

Tabebuia roseoalba (Ridl.) Sandwith

Tecoma stans (L.) Juss. ex Kunth.

Bixaceae

Bixa orelana L.

Ipê-roxo

Jacarandá-de-

minas

$\begin{array}{llllllll}\text { Jacarandá-mimoso } & \mathrm{E} & - & - & 1 & 8 & 9 & 1,18\end{array}$

$\begin{array}{llllllll}\text { Ipê-branco } & \mathrm{N} & 1 & - & - & 1 & 2 & 0,26\end{array}$

Ipê-de-jardim

Caricaceae

Carica papaya L.

Urucum

0,13

Mamão

E 2

Chrysobalanaceae

Licania tomentosa (Benth.) Fritsch

Combretaceae

Terninalia catappa L.

Cordiaceae

Cordia africana Lam.

Cordia trichotoma (Vell.) Arrab. ex

Steud.

Oiti-da-praia

N

$\begin{array}{lll}7 & 12 & 1,58\end{array}$

Chapéu-de-sol

E

0,13

Córdia-africana

E

Louro-pardo

N 1

0,13

Cupressaceae

Cupressus sempervirens $\mathrm{L}$.

Cipreste-do-

mediterrâneo

\section{E}

29

3

32

4,21

\section{Cycadaceae}

Cycas circinalis $\mathrm{L}$.

Cica

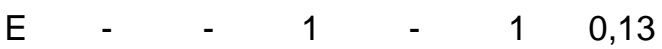

Cycas revoluta Thunb.

\section{Euphorbiaceae}

Euphorbia leucocephala Lotsy

Euphorbia pulcherrima Willd. ex Klotzsch

Cica

E

10

$10 \quad 1,32$

Neve-da-

montanha

Dedália-euphorbia

$\begin{array}{lllllll} & 1 & - & - & - & 1 & 0,13\end{array}$

E

0,13

\section{Fabaceae}

Albizia niopoides (Spruce ex Benth.)

Burkart

Calliandra brevipes Benth.

Cassia fistula L.

Cassia leptophylla Vogel

Cojoba sophorocarpa (Benth.) Britton

\& Rose

Delonix regia (BojerexHook.) Raf.

Enterolobium contortisiliquum (Vell.)

Morong

Erythrina dominguezii Hassl.

Hymenaea courbaril L.

Inga laurina (Sw.) Willd.

Leucaena leucocephala (Lam.) de Wit

Paubrasilia echinata (Lam.) E.Gagnon,

H.C.Lima \& G.P.Lewis

Farinha-seca

Esponja

Cássia-imperial

Falso-barbatimão

Cojoba

Flamboyant

Timburiu

Mulungu

Jatobá

Ingá-de-macaco

Leucena

Pau-Brasil

Peltophorum dubium (Spreng.) Taub.

Phanera variegata (L.) Benth.

Canafístula

Poincianella pluviosa (DC.)

L.P.Queiroz

Pata-de-vaca

Sibipiruna

$\begin{array}{ccccccc}N & - & - & - & 1 & 1 & 0,13 \\ \mathrm{~N} & - & - & 10 & - & 10 & 1,32 \\ \mathrm{E} & - & - & - & 2 & 2 & 0,26 \\ \mathrm{~N} & - & - & - & 1 & 1 & 0,13 \\ \mathrm{E} & - & - & 1 & - & 1 & 0,13 \\ \mathrm{E} & - & 11 & - & 15 & 26 & 3,42 \\ \mathrm{~N} & - & - & 7 & - & 7 & 0,92 \\ \mathrm{~N} & - & - & 2 & - & 2 & 0,26 \\ \mathrm{~N} & 1 & - & 2 & 1 & 4 & 0,53 \\ \mathrm{~N} & - & - & 1 & - & 1 & 0,13 \\ \mathrm{E} & 2 & 1 & - & - & 3 & 0,39 \\ \mathrm{~N} & - & - & 1 & - & 1 & 0,13 \\ \mathrm{~N} & 1 & - & - & - & 1 & 0,13 \\ \mathrm{E} & - & 36 & - & - & 36 & 4,74 \\ \mathrm{~N} & 13 & 20 & 28 & 23 & 84 & 11,0\end{array}$


Senna macranthera (DC. ex Collad.)

H.S.Irwin \& Barneby

Tamarindus indica $\mathrm{L}$.

Tipuana tipu (Benth.) Kuntze

Lamiaceae

Aegiphila integrifolia (Jacq.) Moldenke

Callicarpa reevesii Wall. ex Walp.

Lauraceae

Nectandra megapotamica (Spreng.)

Mez

Persea americana Mill.

\section{Lythraceae}

Lafoensia glyptocarpa Koehne

Lagerstroemia indica $\mathrm{L}$.

Punica granatum $\mathrm{L}$.

\section{Magnoliaceae}

Magnolia champaca (L.) Baill. ex Pierre

\section{Malpighiaceae}

Bunchosia armeniaca (Cav.) DC.

Lophanthera lactescens Ducke

Malpighia emarginata DC.

Malvaceae

Paquira aquatica Aubl.

Paquira glabra Pasq.

\section{Melastomataceae}

Tibouchina granulosa (Desr.) Cogn.

Tibouchina mutabilis (Vell.) Cogn.

\section{Meliaceae}

Cedrela fissilis Vell.

\section{Moraceae}

Artocarpus heterophyllus Lam.

Ficus benjamina L.

Ficus carica L.

Ficus microcarpa L.F.

Maclura tinctoria (L.) D.Don ex Steud.

Morus nigra L.

Myrtaceae

Callistemon viminalis (Sol. ex Gaertn.)

G.Don

Eugenia brasiliensis Lam.

Eugenia involucrata DC.

Eugenia pyriformis Cambess.

Eugenia uniflora L.

$\begin{array}{llllllll}\text { Fedegoso } & \text { N } & 3 & - & - & 2 & 5 & 0,66 \\ \text { Tamarindeiro } & \text { E } & - & 1 & - & - & 1 & 0,13 \\ \text { Tipuana } & \text { E } & - & - & - & 2 & 2 & 0,26 \\ & & & & & & & \\ \text { Tamanqueiro } & \text { N } & 1 & - & - & - & 1 & 0,13 \\ \text { Calicarpa } & \text { E } & - & - & 2 & - & 2 & 0,26\end{array}$

Canelinha

Abacateiro

$\begin{array}{lllllll}\mathrm{N} & - & 1 & 2 & 2 & 5 & 0,66\end{array}$

$\begin{array}{lllllll}\text { E } & 1 & 4 & 7 & - & 12 & 1,58\end{array}$

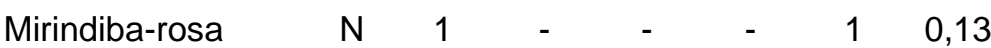

Resedá

E $\quad \begin{array}{lllllc}66 & - & 3 & 7 & 76 & 10,0 \\ \end{array}$

Romanzeiro

E

$\begin{array}{lll}1 & 4 & 0,53\end{array}$

Magnólia-amarela

$E$

Caferana

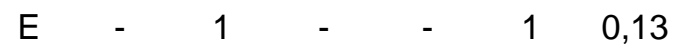

Chuva-de-ouro

Acerola

$N$

E

0,13

0,53

Monguba

Castanha-do-

maranhão

$\begin{array}{lllllll}\mathrm{N} & 2 & 3 & 3 & - & 8 & 1,05\end{array}$

$\mathrm{N}$

0,13

Quaresmeira

$\mathrm{N}$

Manacá-da-serra

N 5

0,66

Cedro-rosa

N

0,13

Jaqueira

Figueira-

benjamina

Figo

Figueira-

lacerdinha

Taiúva

Amoreira-preta

E $\quad-\quad 2$

0,26

E

E

E

N

E

Escova-de-garrafa

Grumixama

Cerejeira-do-rio-

grande

Uvaieira

Pitangueira
E

$\mathrm{N}$

N

N

N 11
2

0,26

0,13

0,13

0,13

1,05 


\begin{tabular}{|c|c|c|c|c|c|c|c|c|}
\hline $\begin{array}{l}\text { Myrciaria glazioviana (Kiaersk.) } \\
\text { G.M.Barroso ex Sobral }\end{array}$ & Cabeludinha & $\mathrm{N}$ & 1 & - & - & - & 1 & 0,13 \\
\hline Plinia cauliflora (Mart.) Kausel & Jabuticabeira & $\mathrm{N}$ & 1 & 1 & - & - & 2 & 0,26 \\
\hline Psidium cattleianum Sabine & Araçá & $\mathrm{N}$ & 1 & - & 1 & - & 2 & 0,26 \\
\hline Psidium guajava L. & Goiabeira & $\mathrm{N}$ & 3 & 10 & - & 4 & 17 & 2,24 \\
\hline Syzygium cumini (L.) Skeels & Jambolão & $E$ & - & 3 & - & - & 3 & 0,39 \\
\hline Syzygium jambos (L.) Alston & Jambo-amarelo & $E$ & - & 3 & - & - & 3 & 0,39 \\
\hline \multicolumn{9}{|l|}{ Oleaceae } \\
\hline Ligustrum lucidum W.T.Aiton & Ligustro & $E$ & - & 5 & - & 1 & 6 & 0,79 \\
\hline \multicolumn{9}{|l|}{ Oxalidaceae } \\
\hline Averrhoa carambola L. & Carambola & $E$ & - & 1 & - & - & 1 & 0,13 \\
\hline \multicolumn{9}{|l|}{ Pinaceae } \\
\hline Pinus oocarpa Schiede ex Schltdl. & Pinheiro-ovo & $E$ & - & - & 5 & - & 5 & 0,66 \\
\hline \multicolumn{9}{|l|}{ Polygonaceae } \\
\hline Triplaris americana L. & Pau-formiga & $\mathrm{N}$ & - & - & 10 & - & 10 & 1,32 \\
\hline \multicolumn{9}{|l|}{ Proteaceae } \\
\hline Grevillea robusta A.Cunn. ex R.Br. & Grevílea & $E$ & 1 & - & 23 & 8 & 32 & 4,21 \\
\hline \multicolumn{9}{|l|}{ Rosaceae } \\
\hline Eriobotrya japonica (Thurn.) Lindl. & Nêspera & $E$ & 1 & - & - & 6 & 7 & 0,92 \\
\hline \multicolumn{9}{|l|}{ Rubiaceae } \\
\hline Coffea arabica L. & Café & $E$ & - & - & - & 4 & 4 & 0,53 \\
\hline \multicolumn{9}{|l|}{ Rutaceae } \\
\hline Citrus medica L. & Limão-cravo & $E$ & 3 & 2 & - & 1 & 6 & 0,79 \\
\hline Citrus sp. & - & $E$ & 1 & 1 & 1 & - & 3 & 0,39 \\
\hline Murraya paniculata (L.) Jack & Murta & $E$ & - & 1 & - & 2 & 3 & 0,39 \\
\hline Zanthoxylum rhoifolium Lam. & Mamica-de-porca & $\mathrm{N}$ & - & 1 & - & - & 1 & 0,13 \\
\hline \multicolumn{9}{|l|}{ Sapindaceae } \\
\hline Sapindus saponaria L. & Saboeiro & $\mathrm{N}$ & - & 1 & - & - & 1 & 0,13 \\
\hline \multicolumn{9}{|l|}{ Sapotaceae } \\
\hline Pouteria caimito (Ruiz \& Pav.) Radlk. & Abieiro & $\mathrm{N}$ & - & 1 & - & - & 1 & 0,13 \\
\hline \multicolumn{9}{|l|}{ Solanaceae } \\
\hline Brunfelsia uniflora (Pohl) D.Don & Manacá-de-cheiro & $\mathrm{N}$ & - & 1 & - & - & 1 & 0,13 \\
\hline Total por praça & & & 208 & 223 & 160 & 169 & & \\
\hline Total & & & & & & & 760 & \\
\hline
\end{tabular}

Legenda: $\mathrm{O}=$ origem; $\mathrm{T}=$ total de indivíduos amostrados; $\mathrm{F}=$ frequência; $\mathrm{N}=$ nativa; $\mathrm{E}$ = exótica; $\mathrm{P} 1$ = Praça Francisco Pinto; P2 = Praça João Paulo II; P3: Praça Ângelo Grizzo; P4 = Praça da Escola Técnica Joaquim Ferreira do Amaral.

Quando comparados os resultados do presente estudo com os de outras praças públicas inventariadas, alguns apresentaram números semelhantes como Sousa et al. (2014) que registraram 36 famílias em uma Praça do Município de São Paulo (SP). No entanto, a maioria dos estudos envolvendo levantamentos florísticos em praças tem registrado número de famílias acentuadamente menor, como é o caso de Assunção et al. (2014) com 12 famílias (Cárceres/MT), Freitas, Pinheiro e Abrahão (2015) com 15 famílias (Bairro da Tijuca/RJ) e Dantas, Gomes e Pinheiro (2016) com apenas 10 famílias (Macapá/AP). 
A família Fabaceae foi a mais representativa com 18 espécies $(16,8 \%)$, seguida por Myrtaceae com 11 espécies (10,2\%), Arecaceae com 10 espécies (9,3\%), Bignoniaceae com 7 espécies (6,5\%) e Moraceae com seis espécies (5,6\%). Essas famílias juntas correspondem a $48,4 \%$ das espécies encontradas, e as demais famílias apresentam cinco espécies ou menos (Tabela 1).

Esses resultados são semelhantes a diversos outros encontrados em estudos realizados pelo Brasil, como é o caso de Redin et al. (2010) para cinco praças no Município de Cachoeira do Sul no Rio Grande do Sul, que também verificaram o predomínio destas famílias na composição florística das praças. Assunção et al. (2014), para quatro praças do Município de Cárceres, no Mato Grosso, também relataram a maior riqueza destas famílias, mesmo com a riqueza de espécies encontrada por eles sendo acentuadamente menor que a do presente estudo. Para o Estado do Rio de Janeiro, Freitas, Pinheiro e Abrahão (2015) evidenciaram as famílias Fabaceae, Arecaceae e Bignoniaceae como as mais diversas. Estas mesmas famílias, também são citadas para três praças analisadas no Município de Leme, Estado de São Paulo (TISCHER; FORTE; PEDROSO-DE-MORAES, 2014). O predomínio destas famílias na flora urbana pode estar relacionado, em parte, com a grande riqueza destas no país, como é o caso evidenciado por BGF (2015) de Fabaceae e Myrtaceae. Além disso, algumas espécies de Arecaceae (Palmeiras imperiais, Palmeira-leque-da-china) e Bignoniaceae (Ipês), apresentam alto potencial paisagístico.

As quatro praças amostradas em Jaú também surpreenderam quanto ao número de espécies encontradas (107), o qual se mostrou amplamente maior que o encontrado em vários outros estudos conduzidos em situações similares a este, como é o caso de Schallenberger et al. (2010) que inventariaram parques e praças e registraram apenas 37 espécies para o Município de Irati (PR). Além desse, Romani et al. (2012) registraram 42 espécies para uma praça no Município de Ribeirão Preto (SP), Assunção et al. (2014) encontraram 25 espécies para quatro praças em Cárceres (MT), Tischer, Forte e Pedroso-de-Moraes (2014), para o Município de Leme (SP), registraram 62 espécies em três praças, e Gomes et al. (2016) encontraram apenas 24 espécies para uma praça em Macapá (AP).

Para vias públicas, segundo Milano e Dalcin (2000), entre 10 e 20 espécies seriam suficientes para a composição destas áreas. O número encontrado no presente estudo foi muito superior ao sugerido por estes autores e por se tratarem de praças, onde, na maioria das vezes, não há conflitos com a fiação, calçadas e redes subterrâneas de água e esgoto, a alta riqueza de espécies pode trazer vários benefícios para a população, bem como para a fauna que habita os centros urbanos.

Apenas três espécies foram encontradas nas quatro praças analisadas, Eugenia uniflora, Morus nigra e Poincianella pluviosa (Tabela 1). Por sua vez, 52 espécies ocorreram 
em apenas uma praça, além disso, muitas espécies apresentaram distribuição irregular dos indivíduos, ou seja, muitos indivíduos ocorreram em uma praça, enquanto nas demais foram registrados poucos indivíduos, por exemplo, Lagerstroemia indica com 66 indivíduos na Praça Francisco Pinto (P1), ausente na Praça João Paulo II (P2) e apenas três e sete indivíduos nas Praças Ângelo Grizzo (P3) e da Escola Técnica Joaquim Ferreira do Amaral (P4), respectivamente (Tabela 1 ).

Vários fatores podem estar relacionados a esta ausência de uniformidade na distribuição das espécies nas praças urbanas de Jaú, dentre eles: a época na qual as praças foram elaboradas, sendo, geralmente, implantadas espécies disponíveis e de interesse paisagístico para a época; a retirada de indivíduos, muitas vezes sem critério, com a intenção de satisfazer projetos da Secretaria de Meio Ambiente, como tem sido observado no município nos últimos anos; e o plantio de novas árvores pelos moradores locais que, apesar de muitas vezes bem intencionados, podem trazer danos ou causar prejuízos, uma vez que não são habilitados para tal função.

Quanto à frequência, a espécie Poincianella pluviosa corresponde a $11,5 \%$ dos indivíduos amostrados, seguida por Lagerstroemia indica com 10\%, Grevillea robusta e Cupressus sempervirens, ambas com 4,41\% e Rostoynea oleracea com 4,08\%, as demais espécies apresentaram menos de $3,5 \%$ de frequência. A presença destas espécies, principalmente Poincianella pluviosa (Sibipiruna), Lagerstroemia indica (Resedá) e Rostoynea oleracea (Palmeira-imperial) entre as mais frequentes, têm sido averiguada em diversas outras áreas do Brasil, como evidenciado por Richter et al. (2012) e Tischer, Forte e Pedroso-deMoraes (2014), o que pode estar relacionado à maior facilidade na obtenção de mudas dessas espécies e à boa adaptação dessas ao ambiente urbano.

Segundo Santamour Júnior (1990), uma família deve apresentar no máximo 30\% das espécies empregadas em uma determinada área arborizada, até 20\% devem pertencer a um mesmo gênero e 10\% a uma mesma espécie. Desta maneira, é possível observar que algumas espécies (Poincianella pluviosa e Lagerstroemia indica) apresentam índices acima dos propostos, o que pode vir a ser um problema em casos de doenças ou pragas nestas áreas.

Quando as praças são analisadas individualmente e comparadas com os índices propostos por Santamour Júnior (1990), observa-se que as famílias e gêneros não apresentaram números próximos ou superiores a estes, mas várias espécies apresentaram números bem acima do desejável. Em P1, Lagerstroemia indica apresenta $31,7 \%$ dos indivíduos. Para P2, Phanera variegata apresenta 16,1\% dos indivíduos e Cupressus sempervirens (com 13\%). Já em P3, Poincianella pluviosa conta com 17,5\% e Grevillea robusta com 14,3\% dos indivíduos. E em P4, Roystonea borinquena apresenta 10,65\% e P. pluviosa $13,6 \%$ dos indivíduos. Isso acaba sendo mais preocupante pois são áreas pequenas e, caso 
uma doença ou praga surja, ela provavelmente se propagará com rapidez devido à elevada taxa de indivíduos da mesma espécie. Por isso, nestas circunstâncias, caso algum sinal de praga ou doença seja detectado, deve ser combatido imediatamente, evitando assim que se propague, comprometendo, desta maneira, o menor número de indivíduos possível.

Dentre as espécies encontradas, 49 (46\%) delas são consideradas nativas do Brasil, enquanto 58 (54\%) são exóticas (Tabela 1), sendo estes números semelhantes aos resultados encontrados em outros estudos (LINDENMAIER; SANTOS, 2008; ROMANI et al., 2012; FREITAS; PINHEIRO; ABRAHÃO, 2015; DANTAS; GOMES; PINHEIRO, 2016). O predomínio de espécies exóticas pode estar relacionado com os atributos de beleza mais valorizados em detrimento das nativas, bem como tendências paisagísticas, mas também pelo desconhecimento da população e dos responsáveis governamentais pela arborização urbana, pela dificuldade em se obter mudas de espécies nativas, principalmente da região (LINDENMAIER; SANTOS, 2008; BRITO et al., 2012), e por essas espécies não apresentarem predadores nem parasitas locais, o que aumenta a chance de sobrevivência.

Além disso, espécies exóticas podem trazer malefícios para a biodiversidade local, sendo uma das principais causas de extinção de espécies, podendo causar danos econômicos (BLUM; BORGO; SAMPAIO, 2008). Algumas das espécies exóticas podem ser consideradas invasoras, tais como Leucaena leucocephala e Tecoma stans, esta última frequentemente observada nos fragmentos florestais do Município de Jaú.

\section{CONCLUSÕES}

As quatro praças analisadas no Município de Jaú apresentam alta riqueza de espécies, 107 espécies no total, bem como um número elevado de indivíduos (760), sendo 208 na Praça Francisco Pinto, 223 na Praça João Paulo II, 160 na Praça Ângelo Grizzo e 169 na Praça da Escola Técnica Joaquim Ferreira do Amaral. Porém foi possível averiguar que algumas poucas espécies, tais como Poincianella pluviosa e Lagerstroemia indica, predominam nestas áreas, sendo este um possível problema caso pragas ou doenças ocorram nestes pontos.

Além disso, mais da metade das espécies (54\%) das áreas inventariadas eram exóticas, o que também pode trazer diversos malefícios, como a diminuição da biodiversidade local, uma vez que essas espécies geralmente não apresentam predadores nem parasitas, o que aumenta a competitividade e a chance de sobrevivência delas.

Com relação ao número de famílias, as quatro praças apresentam maior número em relação ao observado em outros levantamentos em praças. Fabaceae, Myrtaceae, Arecaceae e 
Bignoniaceae foram as mais representativas, assim como observado em outros estudos em praças realizados no Brasil, o que pode estar relacionado à riqueza de espécies e ao potencial paisagístico dessas famílias.

\section{REFERÊNCIAS}

ASSUNÇÃO, K. C.; LUZ, P. B.; NEVES, L. G.; SOBRINHO, P. V. Levantamento quantitativo da arborização de praças da cidade de Cárceres/MT. Revista da Sociedade Brasileira de Arborização Urbana, Piracicaba, v. 9, n. 1, p. 123-132, 2014.

ALVARES, A. C.; STAPE, J. L.; SENTELHAS, P. C.; GONÇALVES, J. L. M.; SPAROVEK, G. Köppen's climate classification map for Brazil. Meteorologische Zeitschrift, Germany, v. 22, n. 6, p. 711-728, 2014.

APG IV. An update of the Angiosperm Phylogeny Group classification for orders and families of flowering plants. Botanical Journal of Linnean Society, London, v. 181, n. 1, p. 1-20, 2016.

BGF. Growing knowledge: an overview of seed plant diversity in Brazil. Rodriguésia, Rio de Janeiro, v. 66, n. 4, p. 1085-1113, 2015.

CARCERERI, V. H.; BIONDI, D.; BATISTA, A. C. Análise da cobertura arbórea das praças de Curitiba - PR. Revista da Sociedade Brasileira de Arborização Urbana, Piracicaba, v. 11, n. 2, p. 12-26, 2016.

COLETO, E. P.; MÜLLER, N. G.; WOLSKI, S. S. Diagnóstico da arborização das vias públicas do município de Sete de Setembro - RS. Revista da Sociedade Brasileira de Arborização Urbana, Piracicaba, v. 3, n. 2, p. 110-122, 2008.

BLUM, C. T.; BORGO, M.; SAMPAIO, A. C. F. Espécies exóticas invasoras de vias públicas de Maringá - PR. Revista da Sociedade Brasileira de Arborização Urbana, Piracicaba, v. 3, n. 2, p. 78-97, 2008.

BRITO, D. R. S.; RAABE, J.; SOUSA, W. C.; MELO, R. R.; PEDROSA, D. C. Diagnóstico da arborização das praças públicas no município de Bom Jesus, Piauí. Scientia Plena, Sergipe, v. 8, n. 4, p. 1-6, 2012.

CHRISTENHUSZ, M. J. M.; REVEAL, J. L.; FARJON, A.; GARDNER, M. F.; MILL, R. R.; CHASE, M. W. A new classification and linear sequence of extant gymnosperms. Phytotaxa, New Zealand, v. 19, p. 55-70, 2011.

DANTAS, A. R.; GOMES, E. M. C.; PINHEIRO, A. P. Diagnóstico florístico da praça Floriano Peixoto na cidade de Macapá, Amapá. Revista da Sociedade Brasileira de Arborização Urbana, Piracicaba, v. 11, n. 2, p. 32-46, 2016.

FREITAS, W. K.; PINHEIRO, M. A. S.; ABRAHÃO, L. L. F. Análise da arborização de quatro praças no Bairro da Tijuca, RJ, Brasil. Floresta e Ambiente, Seropédica, v. 22, n. 1, p. 23-31, 2015.

GOMES, E. M. C.; RODRIGUES, D. M. S.; SANTOS, J. T.; BARBOSA, E. J. Análise qualiquantitativa da arborização de uma praça urbana do norte do Brasil. Nativa, Sinop, v. 4, n. 3, p. 179-186, 2016. 
LINDENMAIER, D. S.; SANTOS, N. O. Arborização urbana das praças de Cachoeira do Sul RS - Brasil: Fitogeografia, diversidade e índice de áreas verdes. Pesquisas, Botânica, São Leopoldo, n. 59, p. 307-320, 2008.

LISTA DE ESPÉCIES DA FLORA DO BRASIL. Jardim Botânico do Rio de Janeiro. Disponível em: <http://floradobrasil.jbrj.gov.br/> Acesso em: 15mar. 2017.

MILANO, M.; DALCIN, E. Arborização de vias públicas. Rio de Janeiro: Light, 2000. 226p.

MOBOT. Missouri Botanical Garden. Disponível em: <http://tropicos.org> Acesso em: 15mar. 2017.

REDIN, C. G.; VOGEL, C.; TROJAHN, C. D. P.; GRACIOLI, C. R.; LONGHI, S. J. Análise da arborização urbana em cinco praças do município de Cachoeira do Sul, RS. Revista da Sociedade Brasileira de Arborização Urbana, Piracicaba, v. 5, n. 3, p. 149-164, 2010.

RICHTER, C.; PEITER, M. X.; ROBAINA, A. D.; SOUZA, A .R. C.; FERRAZ, R. C.; DAVID, A. F. Levantamento da arborização urbana pública de Mata/RS. Revista da Sociedade Brasileira de Arborização Urbana, Piracicaba, v. 7, n. 3, p. 88-96, 2012.

ROBBA, F.; MACEDO, S. S. Praças brasileiras. São Paulo: EDUSP, 2010.

ROMANI, G. N.; GIMENES, R.; SILVA, M. T.; PIVETTA, K. F. L.; BATISTA, G. S. Análise qualiquantitativa da arborização da praça XV de Novembro em Ribeirão Preto - SP, Brasil. Revista Arvore, Viçosa, v. 36, n. 3, p. 479-487, 2012.

SANTAMOUR JÚNIOR, F. S. Trees for urban planting: diversity uniformity, and common sense. In: METRIA CONFERENCE, 7., 1990, Lisle. Proceedings... Lisle: Metria Conference, 1990. p. 57-66.

SCHALLENBERGER, L. S.; ARAUJO, A. J.; ARAUJO, M. N.; DEINER, L. J.; MACHADO, G. O. Avaliação da condição de árvores urbanas nos principais parques e praças do município de Irati-PR. Revista da Sociedade Brasileira de Arborização Urbana, Piracicaba, v. 5, n. 2, p. 105-123, 2010.

SILVA, M. D. M.; SILVEIRA, R. R.; TEIXEIRA, M. I. J. G. Avaliação da arborização de vias públicas de uma área da região oeste da cidade de Franca/SP. Revista da Sociedade Brasileira de Arborização Urbana, Piracicaba, v. 3, n. 1, p. 19-35, 2008.

SOUSA, R. C.; AGUIAR, O. T.; SILVA, L. T. A.; SILVA, L. A.; MARRA, R. C. Avaliação qualiquantitativa da arborização na praça Agostinho Nohama, bairro Lauzane Paulista, São Paulo SP. Revista da Sociedade Brasileira de Arborização Urbana, Piracicaba, v. 9, n. 1, p. 92107, 2014.

TISCHER, J. C.; FORTE, A. R.; PEDROSO-DE-MORAES, C. Análise qualiquantitativa de indivíduos arbóreos das praças centrais do município de Leme, SP. Revista da Sociedade Brasileira de Arborização Urbana, Piracicaba, v. 9, n. 3, p. 49-94, 2014. 